\title{
A review on COVID-19 forecasting models
}

\author{
Iman Rahimi ${ }^{1} \cdot$ Fang Chen ${ }^{2} \cdot$ Amir H. Gandomi ${ }^{2}$ (D)
}

Received: 16 September 2020 / Accepted: 11 December 2020

(C) The Author(s), under exclusive licence to Springer-Verlag London Ltd. part of Springer Nature 2021

\begin{abstract}
The novel coronavirus (COVID-19) has spread to more than 200 countries worldwide, leading to more than 36 million confirmed cases as of October 10, 2020. As such, several machine learning models that can forecast the outbreak globally have been released. This work presents a review and brief analysis of the most important machine learning forecasting models against COVID-19. The work presented in this study possesses two parts. In the first section, a detailed scientometric analysis presents an influential tool for bibliometric analyses, which were performed on COVID-19 data from the Scopus and Web of Science databases. For the above-mentioned analysis, keywords and subject areas are addressed, while the classification of machine learning forecasting models, criteria evaluation, and comparison of solution approaches are discussed in the second section of the work. The conclusion and discussion are provided as the final sections of this study.
\end{abstract}

Keywords Forecasting · Analysis · COVID-19 · SIR · SEIR · Time series

\section{Introduction}

In December 2019, the Chinese government informed the rest of the world that a novel coronavirus, Severe Acute Respiratory Syndrome-Related Coronavirus 2 (COVID19), was rapidly spreading throughout China, which quickly infiltrated many other countries. The United States Centers for Disease Control and Prevention (CDC) recognized a seafood market in Wuhan as the center of the outbreak. On January 13, 2020, the World Health Organization (WHO) reported a case in Thailand, the first case to be identified outside China. On January 16, Japan confirmed its first case, and on January 20, South Korea reported its first confirmed case. Nowadays, most countries in the world have been affected by this virus.

Putra and Khozin Mu'tamar [1] used the Particle Swarm Optimization (PSO) algorithm to estimate parameters in the Susceptible, Infected, Recovered (SIR) model. The

Amir H. Gandomi

gandomi@uts.edu.au

1 Department of Mechanical and Manufacturing Engineering, Faculty of Engineering, Universiti Putra Malaysia, Seri Kembangan, Malaysia

2 Data Science Institute, University of Technology Sydney, Ultimo 2007, NSW, Australia results indicate that the suggested method is precise and has low enough error compared to other analytical methods. Mbuvha and Marwala [2] calibrated the SIR model to South Africa's reported cases after considering different scenarios of the reproduction number (R0) for reporting infections and healthcare resource estimations. Qi and Xiao [3] proposed that both daily temperature and relative humidity can influence the occurrence of COVID-19 in Hubei and other provinces.

Salgotra and Gandomi [4] developed two COVID-19 prediction models based on genetic programming and applied these models in India. Findings from a study by [4] show that genetic evolutionary programming models have proven to be highly reliable for COVID-19 cases in India.

The rest of paper is organized into the following sections. Sections 2 and 3 present the search method procedure and other reviews, respectively. Section 4 shows the main research fields. Generic illustrations are provided in Sect. 5. Mathematical modeling and criteria evaluation are presented in Sects. 6 and 7. Solution approaches, including autoregressive model, exponential models, deep learning, regression methods, etc., are described in Sect. 8. Section 9 depicts the strengths and weaknesses of the various forecasting models. Finally, the conclusion, discussion of results, and future directions are presented in Sect. 10. 


\section{Search method procedure}

The process used to identify the articles for this study's review is presented in this section.

\subsection{Search method}

Web of Science (WOS) and Scopus were used to find related publications based on the following keywords: forecasting, prediction, COVID-19, and coronavirus. The classification of the chosen published works based on the subject area is displayed in Fig. 1. Updated articles from the beginning of 2020 to now were filtered from Scopus using the Boolean operator OR, for both topics and titles. We selected 920 technical research articles that contain only algorithmic descriptions, review articles, conference papers, case studies, and provide managerial insights, which were published as of October 10, 2020 (Fig. 2). In addition, this study focuses more on those papers that were indexed by the Web of Science.

\section{Other reviews}

Mahalle and Kalamkar [5] categorized forecasting models as mathematical models and machine learning techniques, using WHO and social media communications as datasets. Significant parameters including death count, metrological parameters, quarantine period, medical resources, and mobility were also studied [5].

Naudé [6] provided a review of the contribution of artificial intelligence (AI) against COVID-19. Some fields of AI that have contribution against COVID-19 have been identified as early warnings and alerts, tracking and prediction, data dashboards, diagnosis and prognosis, treatments and cures, and social control [6].

\section{Main research fields}

Keywords are critical in identifying the appropriate literature in a research field [7]. As specified by [8]: "keywords represent the core research of a paper." A keywords
Fig. 1 Classification of scientific papers based on subject area

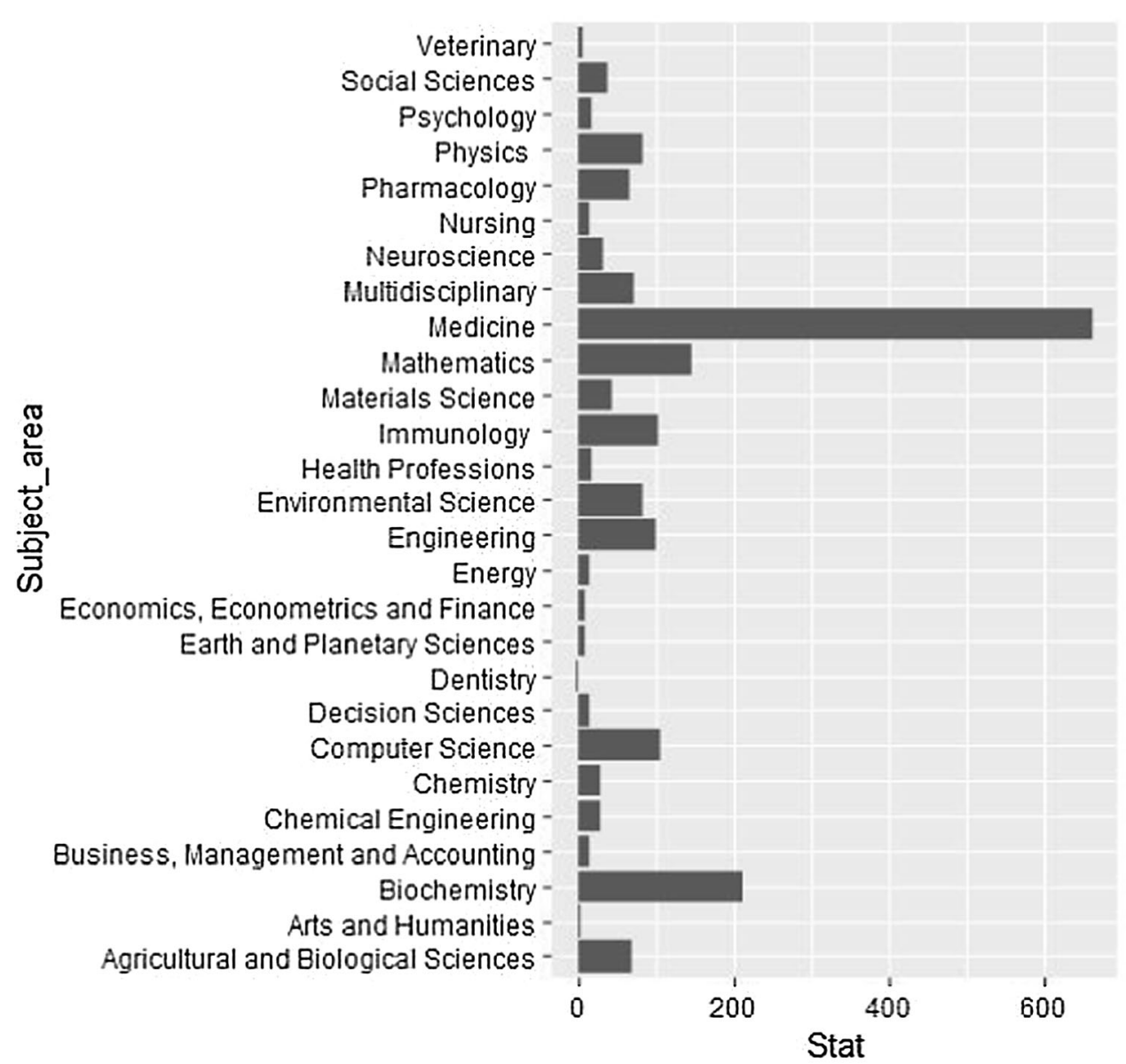


Fig. 2 Research methodology used in this paper

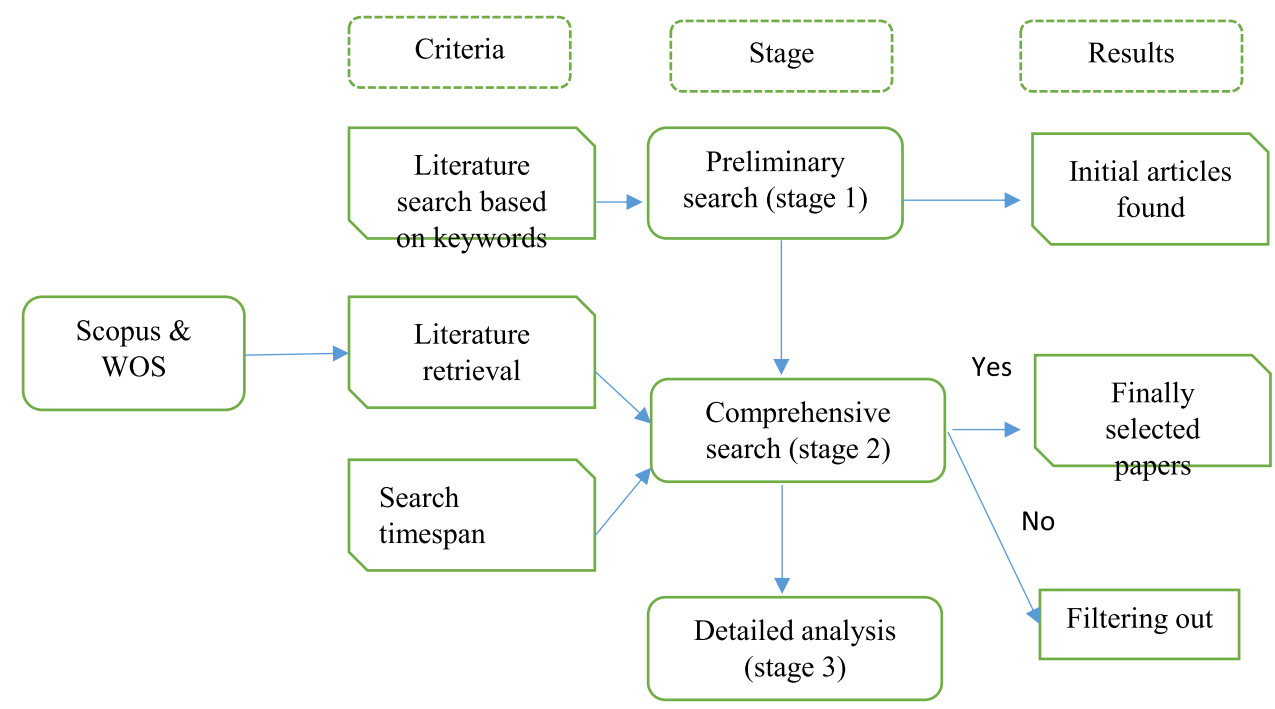

network offers a copy of an information area that provides insight into the available subjects and how these topics are related and sorted [9]. Therefore, the VOSviewer 1.6.11 software was applied to provide a keyword co-occurrence network, and bibliographic data were derived from Scopus. Author keywords were used to generate a network of keywords. A sum of 1931 keywords were obtained from the dataset, regarding the full counting. Table 1 presents the parameter settings for keyword visualization.

The resulting network contains 500 nodes and 4000 links, as shown in Fig. 3, which also presents the main fields for forecasting coronavirus. Stronger links in the network visualization are indicated by thicker lines [10]. It can be seen in Fig. 3 that Coronavirus, prediction, epidemic, human, and forecasting have connection links. Moreover, Fig. 3 presents a network visualization based on keywords, where Coronavirus, prediction, epidemic, human, statistical analysis, quarantine, hospitalization, mortality, and weather are among the top keywords on which researchers focused. In Fig. 3, the cluster is indicated by color, and the bigger circle represents the keyword that is used most.

Figure 4 presents the detailed analysis of the sum of works cited and the number of records versus affiliations. The filtered numbers of records and works cited include a minimum of 1 and 18 , respectively.

Table 1 Parameter settings

\begin{tabular}{ll}
\hline Parameter & Value \\
\hline Minimum number of occurrences & 1 \\
Criterion met & 1931 keywords \\
\hline
\end{tabular}

\section{Generic illustrations}

Several epidemic models have been used by researchers to estimate the outbreak in the short and long term [11-14]. The most applied epidemic models are the susceptible, infected, and recovered (SIR) model and susceptible, exposed, infected, and recovered (SEIR). The SIR model $[15,16]$ is described as shown in Fig. 5:

In terms of mathematical modeling, the SIR model is shown below [17]:

$\frac{\mathrm{d} s}{\mathrm{~d} t}=-\beta I S$

$\frac{\mathrm{d} I}{\mathrm{~d} t}=\beta I S-\gamma I$

$\frac{\mathrm{d} R}{\mathrm{~d} t}=\gamma I$

where $S$ is the number of individuals susceptible at time $t ; I$ is the number of infected individuals at time $t ; R$ is the number of recovered individuals at time $\mathrm{t}$; and $\beta$ and $\gamma$ are the transmission rate and rate of recovery (removal), respectively. The SEIR model [18] is similar to the SIR model except that variable $\mathrm{E}$ is added for the fraction of individuals that have been infected but are asymptomatic. The SEIR model and the related equations are presented in Fig. 6.

The equations of the SEIR model are defined below:

$\frac{\mathrm{d} S(t)}{\mathrm{d} t}=-\beta \frac{S(t) I(t)}{N}-\alpha S(t)$

$\frac{\mathrm{d} E(t)}{\mathrm{d} t}=\beta \frac{S(t) I(t)}{N}-\gamma E(t)$ 


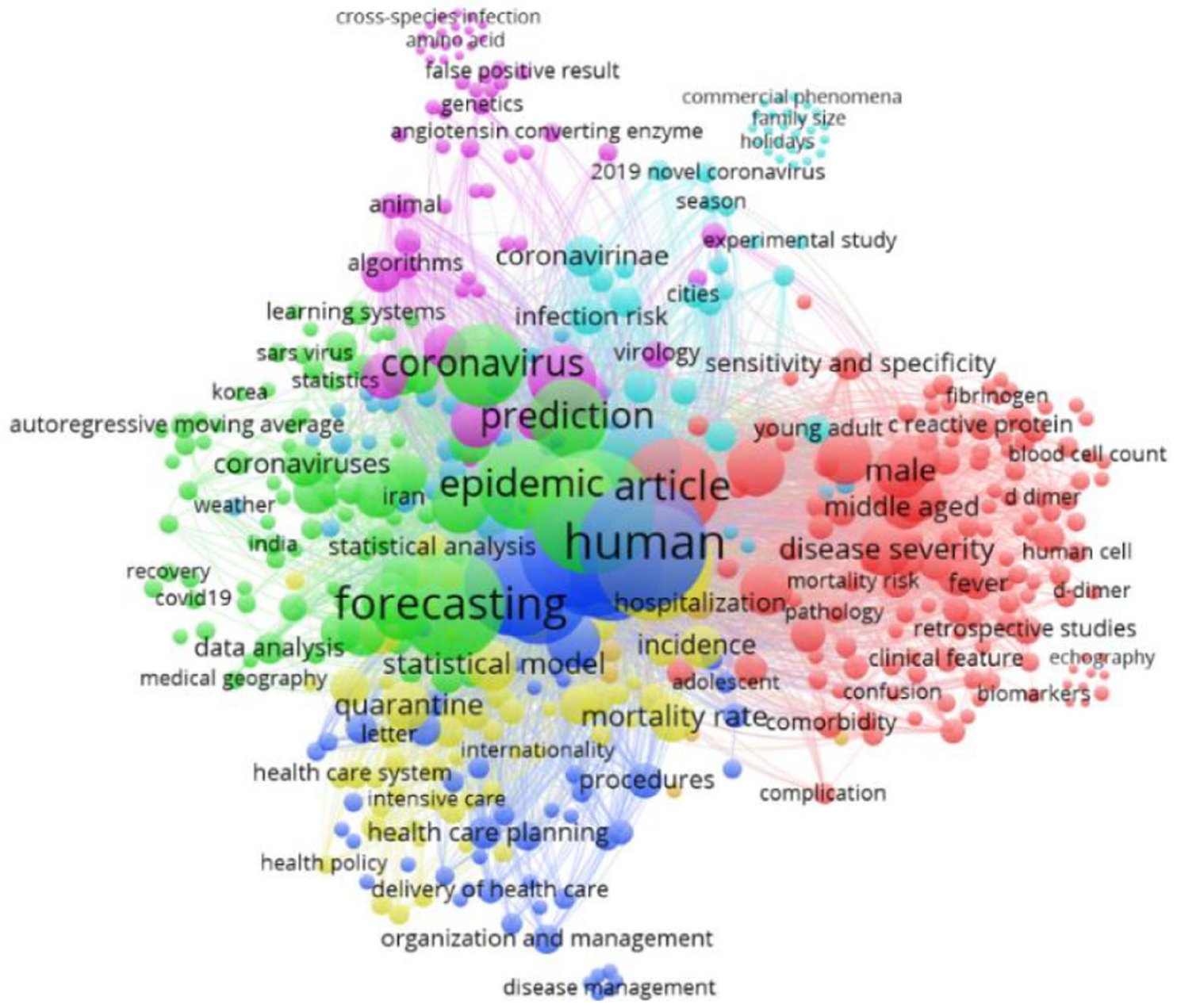

Fig. 3 Networks across the links (keywords analysis)

$$
\begin{aligned}
& \frac{\mathrm{d} I(t)}{\mathrm{d} t}=\gamma E(t)-\delta I(t) \\
& \frac{\mathrm{d} Q(t)}{\mathrm{d} t}=\delta I(t)-\lambda(t) Q(t)-\kappa(t) Q(t) \\
& \frac{\mathrm{d} R(t)}{\mathrm{d} t}=\lambda(t) Q(t) \\
& \frac{\mathrm{d} D(t)}{\mathrm{d} t}=\kappa(t) Q(t) \\
& \frac{\mathrm{d} P(t)}{\mathrm{d} t}=\alpha S(t)
\end{aligned}
$$

where $\alpha$ depicts the protection rate; $\beta$ is the infection rate; $\gamma$ is the inverse of the average latent time; $\delta$ represents the inverse of the average quarantine time; $\lambda_{0}$ and $\lambda_{1}$ are coefficients used in the time-dependent cure rate; and $\kappa_{0}$ and $\kappa_{1}$ are coefficients used in the time-dependent mortality rate [18].

\section{Mathematical modeling}

Ahmar and del Val [19] used the SutteARIMA method to forecast short-term confirmed cases of COVID-19 and Spain Market Index (IBEX 35). Comparatively, the SutteARIMA method was found to be more suitable for forecasting daily confirmed cases in Spain than the AutoRegressive Integrated Moving Average (ARIMA) based on the mean absolute percentage error (MAPE) values. Al-qaness [20] suggested an improved version of the Adaptive Neuro-Fuzzy Inference System (ANFIS) based on the Flower Pollination Algorithm (FPA) by using the Salp Swarm Algorithm to forecast the number of confirmed cases of COVID-19 in China. The idea is to determine the parameters of the Adaptive Neuro-Fuzzy Inference System using the hybrid of the Flower Pollination and Salp Swarm Algorithms. The performance of FPA was validated by comparing it with the existing modified ANFIS models, such as Particle Swarm Optimization (PSO), genetic algorithm (GA), approximate Bayesian 


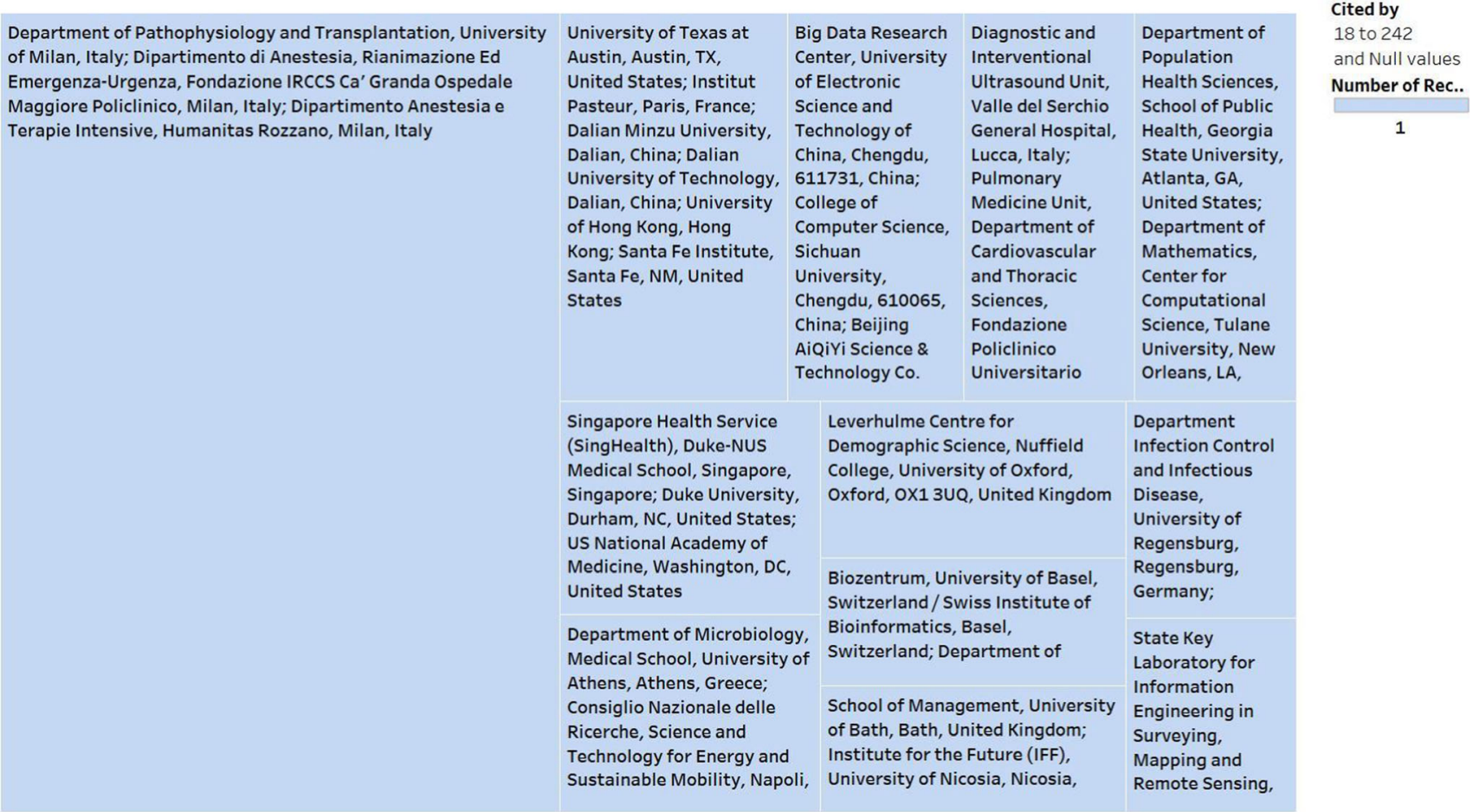

Fig. 4 A detailed analysis (sum of works cited and number of records vs. Affiliations)

\section{Susceptible (S) \\ Infectious (I) \\ Recovered (R)}

Fig. 5 Susceptible, infected, and recovered (SIR) model

computation (ABC), and FPA. Anastassopoulou and Russo [21] proposed a method for predicting the reproduction number (R0) from the susceptible, infected, recovered, and deceased (SIRD) model and other key parameters in forecasting the spread of the COVID-19 epidemic in China. Chakraborty and Ghosh [22] presented a real-time forecast of confirmed COVID-19 cases for multiple countries as well as a risk assessment of the novel COVID-19 for some profoundly affected countries using the regression tree algorithm. A simple moving average approach was used by [23] to predict COVID-19 confirmed cases in Pakistan. [24] used a five-parameter logistic growth model to reconstruct and forecast the COVID-19 epidemic in the USA; however, the authors claimed the accuracy of their model depends on federal- and state-level policy decisions. Cheng and Burcu [12] introduced a platform, icumonitoring.ch, to provide hospital-level projections for intensive care unit (ICU) occupancy based on SEIR models. The proposed platform could help ICU managers to estimate

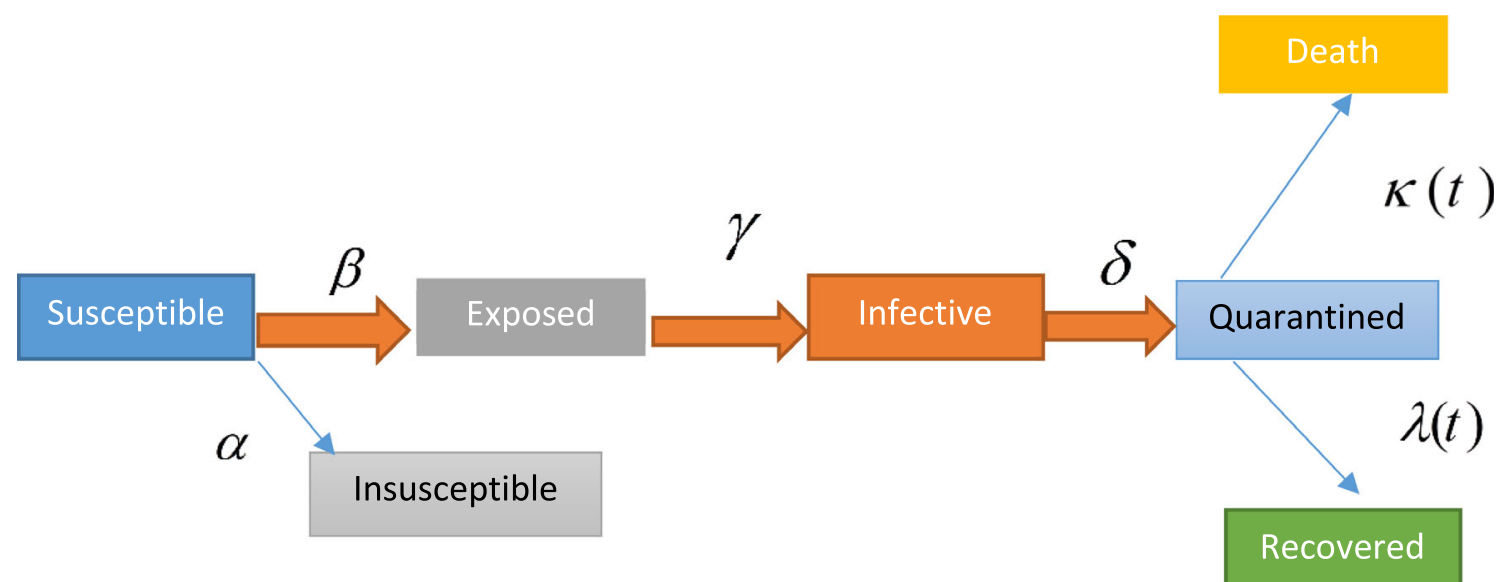

Fig. 6 The susceptible, exposed, infected, and recovered (SEIR) diagram [18] 
the need for additional resources and is updated every 3-4 days. Chimmula and Zhang [25] applied long shortterm memory (LSTM) networks as a deep learning technique for predicting COVID-19 outbreaks in Canada. Their approach identified the key features for estimating the trends of the pandemic in Canada. A simple ARIMA model was proposed by [26] to estimate registered and recovered cases after a lockdown in Italy.

Salgotra and Gandomi [4] established two COVID-19 prediction models based on genetic programming in India. Their results indicate that genetic evolutionary programming models are highly reliable for COVID-19 cases in India. Dil and Dil [11] used the SIR model to forecast confirmed COVID-19 cases in the Eastern Mediterranean region, namely Iran, Iraq, Saudi Arabia, United Arab Emirates, Lebanon, Egypt, and Pakistan, with a special focus on Pakistan. A simple SIRD model was proposed by [14] to predict COVID-19 outbreaks in China, Italy, and France and estimate healthcare facility necessities, such as ventilation units.

\section{Criteria evaluation}

Forecasting confirmed cases, risk assessment, stock market, ICU beds, registered and recovered cases are top criteria in which scholars show heightened interest.

\section{Solution approaches}

Several approaches have been addressed by researchers to predict the COVID-19 outbreak [27, 28]. Table 2 presents the solution approaches proposed by researchers for forecasting COVID-19, among which SIR, SEIR, SIRD, and Moving Average are the most popular approaches. Also, some researchers $[29,30]$ preferred to use hybrid algorithms to enhance the power of forecasting algorithms.

\subsection{Autoregressive model}

The autoregressive time-series model is known as a useful tool to model dependent data and has been applied to various real-world problems [49-53].

\subsubsection{Moving average}

In statistics and economics, a moving average is a way to calculate and analyze data by providing a series of averages of various subsets of the dataset [54].

8.1.1.1 Simple moving average A simple moving average (SMA) is defined as the unweighted mean of the previous data (in finance) or an equal number of data on either side of a central value (in science or engineering) [54]. An example of an application of a simple moving average in COVID-19 could be found in [23].

8.1.1.2 Autoregressive integrated moving average (ARIMA) An autoregressive integrated moving average model is a generalized form of the autoregressive moving average model. As it is well known for forecasting, some researchers have used ARIMA to predict the spread of the new pandemic [31, 55-58].

\subsubsection{Two-piece distributions based on the scale}

Maleki M et al. [35] proposed an autoregressive time-series model based on two-piece scale mixture normal distribution to predict confirmed and recovered COVID-19 cases. Compared with the standard autoregressive time-series model, the proposed algorithm outperforms others in the forecasting the confirmed and recovered COVID-19 cases around the world.

\subsection{Exponential models}

Exponential models are suitable in the modeling of several phenomena, such as populations, interest rates, and infectious diseases [59].

\subsubsection{Logistic functions}

One of the famous S-shaped curves is logistic a function with application in biology, chemistry, linguistics, political science, and statistics. [24, 37, 38] provide examples of applications of logistic functions in COVID-19.

\subsubsection{Deep learning}

Deep learning is a famous branch of machine learning in which the learning process can be supervised, semi-supervised, unsupervised [60-62]. Application of different forms of deep learning in forecasting COVID-19 cases could be found in long short-term memory (LSTM) networks [25, 63], polynomial neural network [39], and neural network $[31,40]$.

\subsubsection{Regression methods}

In statistics, regression methods are a set of statistical modeling to estimate the relationship between a dependent variable and independent variable(s) [64, 65]. As a powerful tool to forecast the pandemic, various regression methods have been addressed by researchers against COVID-19 [42-44, 66, 67]. 
Table 2 Proposed solution approaches for forecasting coronavirus 2019 (COVID-19)

\begin{tabular}{|c|c|c|c|c|c|c|c|c|}
\hline \multicolumn{9}{|l|}{ Algorithm } \\
\hline \multicolumn{2}{|l|}{ Epidemic models } & \multicolumn{6}{|l|}{ Time-series models } & \multirow{3}{*}{$\begin{array}{l}\text { Nature-inspired } \\
\text { algorithms } \\
\text { Genetic } \\
\text { programming } \\
\text { [30-34] }\end{array}$} \\
\hline \multirow[t]{3}{*}{ SIR } & \multirow[t]{3}{*}[11]{} & \multirow[t]{3}{*}{$\begin{array}{l}\text { Autoregressive } \\
\text { model }\end{array}$} & \multirow[t]{2}{*}{ Moving average } & \multicolumn{4}{|c|}{$\begin{array}{l}\text { Autoregressive integrated moving average } \\
{[19,22,26,30,31]}\end{array}$} & \\
\hline & & & & \multicolumn{4}{|c|}{ Simple moving average [23] } & \\
\hline & & & Other models & \multicolumn{4}{|l|}{$[35]$} & \\
\hline SEIR & {$[12,36]$} & \multicolumn{2}{|l|}{ Exponential models } & \multicolumn{4}{|c|}{ Logistic growth model $[24,37,38]$} & $\begin{array}{l}\text { Flower } \\
\text { pollination } \\
\text { algorithm } \\
\text { [29] }\end{array}$ \\
\hline \multirow[t]{4}{*}{ SIRD } & \multirow[t]{4}{*}[13,14]{} & Deep learning & \multicolumn{5}{|c|}{ Long short-term memory (LSTM) networks [25] } & $\begin{array}{l}\text { Polynomial } \\
\quad \text { Neural } \\
\text { Network [39] }\end{array}$ \\
\hline & & & \multicolumn{5}{|c|}{ Neural network $[31,40]$} & Ecological \\
\hline & & $\begin{array}{l}\text { Regression } \\
\text { methods }\end{array}$ & \multicolumn{5}{|l|}{ [42-44] } & $\begin{array}{l}\text { Niche models } \\
\text { [41] }\end{array}$ \\
\hline & & Prophet algorithm & \multicolumn{5}{|l|}{$[45]$} & \\
\hline $\begin{array}{l}\text { Phenomenological } \\
\text { model }\end{array}$ & {$[46]$} & Other models & $\begin{array}{l}\text { Adaptive neuro- } \\
\text { fuzzy } \\
\text { inference } \\
\text { system [29] }\end{array}$ & $\begin{array}{l}\text { Regression } \\
\text { tree } \\
\text { algorithm } \\
{[22]}\end{array}$ & $\begin{array}{l}\text { Support } \\
\text { vector } \\
\text { machine } \\
{[39,47]}\end{array}$ & $\begin{array}{l}\text { Iteration } \\
\text { method } \\
{[48]}\end{array}$ & $\begin{array}{r}\text { Support } \\
\text { vector } \\
\text { Kuhn- } \\
\text { tucker } \\
\text { [47] }\end{array}$ & \\
\hline
\end{tabular}

\subsubsection{Prophet algorithm}

The Prophet algorithm is an open-source tool that works well with time-series data that have seasonal effects. The main goal of the algorithm, developed by Facebook's Data Science team, is business forecasting [68, 69]. The Prophet algorithm has proven to be robust in dealing with missing data [70].

\subsubsection{Genetic programming}

Genetic programming (GP) is a nature-inspired algorithm, where the keys include program representation (tree structure), selection, crossover, and mutation [71]. Some examples of GP in COVID-19 are available in [32-34].

\subsubsection{SIR}

One of the most applied epidemic models is the susceptible, infected, and recovered (SIR) model $[15,16]$. Variables S, I, and R are defined in Eqs. 1-3.

\subsubsection{SEIR}

The SEIR model [18] is an extended version of the SIR model, which considers an additional parameter, $E$, representing the fraction of individuals that have been infected but are asymptomatic.

\subsubsection{SIRD}

The SIRD model differentiates between recovered individuals (those who have survived the disease and are now immune) and deceased individuals [13, 14].

\section{Strengths and weaknesses of forecasting models}

As discussed earlier, many machine learning algorithms have been used to forecast the new pandemic in different places of the world. Figure 7 presents the percentage of contribution of different solution approaches applied in forecasting COVID-19 confirmed cases (there are 925 indexed articles in Scopus as of October 10, 2020). As it is clear from Fig. 7, deep learning, compartmental models, and other methods have the most contributions, while the Prophet algorithm, as a new branch of machine learning, has the least contribution.

Machine learning algorithms exhibit many pros and cons, which are described in Table 3. 
Fig. $7 \%$ of contribution of different solution approaches applied in the forecasting of COVID-19 confirmed cases

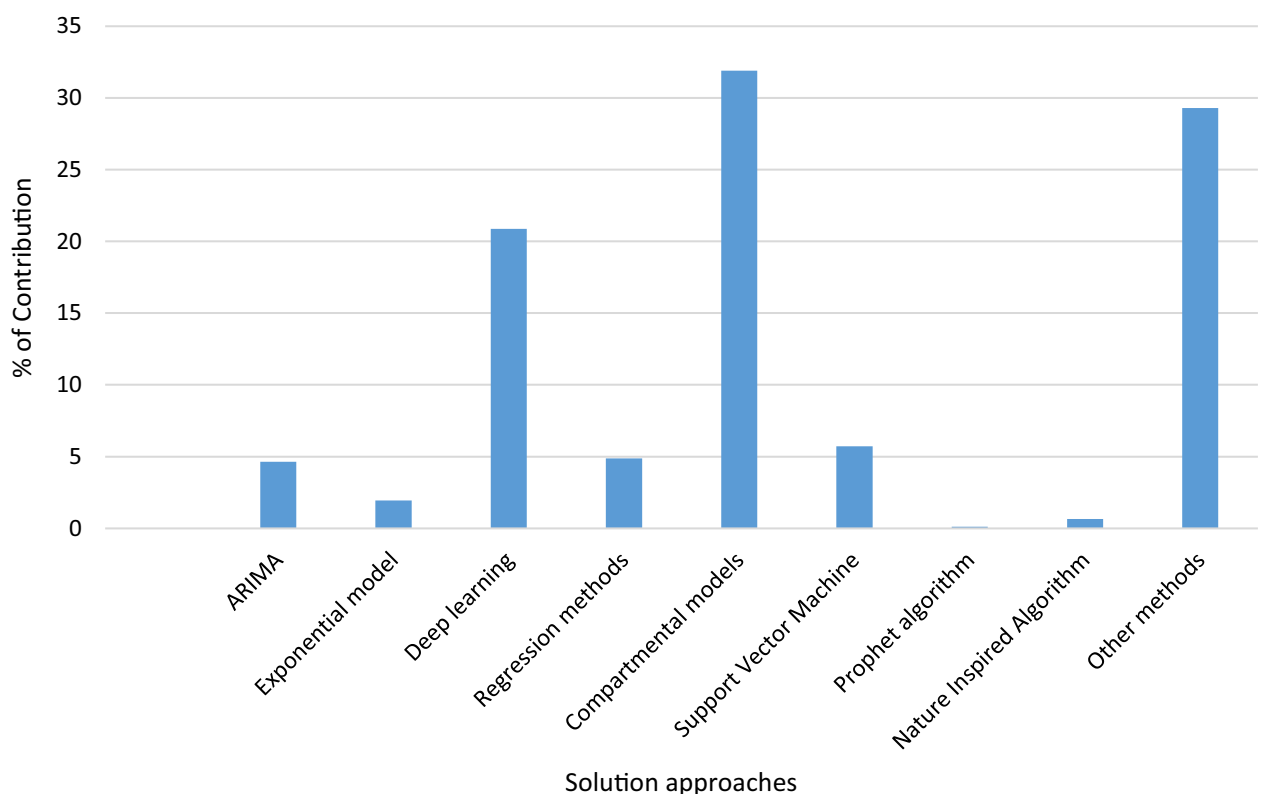

\section{Conclusion and discussion}

At the time of writing, COVID-19 had spread to more than 200 countries worldwide with more than 36 million confirmed cases. Several works have been released in the field for predicting global outbreaks. This study aimed to review the most important forecasting models for COVID-19 and provides a short analysis of published literature. This paper highlighted the most important subject areas by keywords analysis. Moreover, several criteria were identified that could help researchers for future works. Also, this paper recognized the most useful models that researchers have applied for predicting this pandemic. Furthermore, this paper may help researchers to identify important gaps in the research area and, subsequently, develop new machine learning models for forecasting the COVID-19 cases. A detailed scientometric analysis was performed as an influential tool for use in bibliometric analyses and reviews. For this aim, keywords and subject areas are discussed, while

Table 3 Strengths and weaknesses of proposed machine learning algorithms

\begin{tabular}{|c|c|c|}
\hline Algorithm & Strength & Weakness \\
\hline Artificial neural network & Could access several training algorithms [72] & The nature of being a black box [72], overtraining [73] \\
\hline Support vector machine & $\begin{array}{l}\text { Can avoid overfitting and defining a convex } \\
\text { optimization problem [72] }\end{array}$ & $\begin{array}{l}\text { Choice of the kernel as well as speed and size of } \\
\text { training and testing sets [72] }\end{array}$ \\
\hline $\begin{array}{l}\text { Compartmental models (SIR, } \\
\text { SEIR, SIRD, etc.) }\end{array}$ & $\begin{array}{l}\text { Predict how the disease spreads } \\
\text { Present the effects of public health interventions on } \\
\text { the outcome of the pandemic [74-78] }\end{array}$ & $\begin{array}{l}\text { The proposed models are mostly deterministic and } \\
\text { work with large populations [79] }\end{array}$ \\
\hline $\begin{array}{l}\text { Nature-inspired algorithms } \\
\text { (genetic programming) }\end{array}$ & $\begin{array}{l}\text { Intelligent search }[80] \\
\text { Can integrate with certain decomposition algorithms } \\
\text { [81] }\end{array}$ & $\begin{array}{l}\text { Several parameters should be set by the decision- } \\
\text { makers } \\
\text { The algorithms are approximate and usually } \\
\text { nondeterministic [82] }\end{array}$ \\
\hline Prophet algorithm & Are robust in dealing with missing data [70] & $\begin{array}{l}\text { It is hard to use the algorithm for Multiplicative } \\
\text { models } \\
\text { Predefined format is needed for data before using the } \\
\text { algorithm }\end{array}$ \\
\hline ARIMA & $\begin{array}{l}\text { Works for seasonal and nonseasonal models } \\
\text { Outliers can be handled well }\end{array}$ & $\begin{array}{l}\text { Changes in observations and changes } \\
\text { in model specification make the model unstable [83] }\end{array}$ \\
\hline Deep learning & $\begin{array}{l}\text { Results comparable to human expert performance } \\
{[84,85]}\end{array}$ & $\begin{array}{l}\text { Requires large amounts of data } \\
\text { The training process is expensive }\end{array}$ \\
\hline
\end{tabular}


the classification of forecasting models, criteria evaluation, and comparison of solution methods are provided in the second section of the work.

This study describes some key arguments that are worthy of further discussion:

- In terms of the subject area, medicine, biochemistry, and mathematics are most discussed areas addressed by scholars.

- In terms of keywords analysis, trends present that studies on COVID-19 will increase in the next few months. Moreover, Coronavirus, prediction, epidemic, human, statistical analysis, quarantine, hospitalization, mortality, and weather instances are the most interesting keywords for scholars.

- Several other criteria have been used by researchers in forecasting, including:

- Confirmed cases, risk assessment, stock market, ventilation units, ICU beds, estimated registered and recovered cases.

- Several countries, including China, Pakistan, France, Italy, USA, UK, Brazil, Nigeria, Iran, Germany, and India, were addressed as case studies.

- Among the epidemic models, deep learning, SIR, and SEIR are the top models that were used by researchers.

- Hybrid algorithms are used to enhance the power of forecasting approaches.

- The majority of studies are deterministic approaches, while there is an urgent need to provide robust approaches for tackling uncertain situations.

For future research directions, a comprehensive review in other fields, such as artificial intelligence (AI) and deep learning, is encouraged. Moreover, more studies addressing the development of novel and hybrid approaches to forecast the pandemic should be investigated. Furthermore, at the time of writing this paper, we had access to only a limited number of published articles by Scopus and WOS. However, the most important parts of this paper are the keywords and scientometric analysis that consider the whole database, from which we chose some examples of published articles for review. Therefore, a more comprehensive review in the research area is suggested.

Funding The authors confirm that there is no source of funding for this study.

\section{Compliance with ethical standards}

Conflict of interest The authors declare that they have no conflict of interest.

\section{References}

1. Putra S, Khozin Mutamar Z (2019) Estimation of parameters in the SIR epidemic model using particle swarm optimization. Am J Math Comput Model 4(4):83-93

2. Mbuvha RR, Marwala T (2020) On data-driven management of the COVID-19 outbreak in South Africa. medRxiv

3. Qi H et al (2020) COVID-19 transmission in Mainland China is associated with temperature and humidity: a time-series analysis, p 138778

4. Salgotra R, Gandomi M, Gandomi AH (2020) Time series analysis and forecast of the COVID-19 pandemic in india using genetic programming. Chaos Solitons Fractals 138:109945

5. Mahalle $\mathrm{P}$ et al (2020) Forecasting models for coronavirus (covid-19): a survey of the state-of-the-art. TechRxiv. https://doi. org/10.36227/techrxiv.12101547.v1 (Preprint)

6. Naudé W (2020) Artificial intelligence against COVID-19: an early review. IZA Discussion Paper No. 13110. https://ssrn.com/ abstract $=3568314$

7. Shrivastava R, Mahajan PJS, Libraries T (2016) Artificial intelligence research in India: a scientometric analysis. Sci Technol Libr 35(2):136-151

8. Su H-N, Lee P-CJS (2010) Mapping knowledge structure by keyword co-occurrence: a first look at journal papers in technology foresight. Scientometrics 85(1):65-79

9. Van Eck NJ, Waltman L (2014) Visualizing bibliometric networks. In: Ding Y, Rousseau R, Wolfram D (eds) Measuring scholarly impact. Springer, Berlin, pp 285-320

10. Van Eck NJ, Waltman L (2013) VOSviewer manual, vol 1. Univeristeit Leiden, Leiden, pp 1-53

11. Dil S, Dil N, Maken ZH (2020) COVID-19 trends and forecast in the eastern mediterranean region with a particular focus on Pakistan. Cureus 12(6):8

12. Cheng $Z$ et al (2020) icumonitoring.ch: a platform for short-term forecasting of intensive care unit occupancy during the COVID19 epidemic in Switzerland. Swiss Med Weekly 150:10

13. Anastassopoulou C et al (2020) Data-based analysis, modelling and forecasting of the COVID-19 outbreak. PLoS ONE 15(3):21

14. Fanelli D, Piazza F (2020) Analysis and forecast of COVID-19 spreading in China, Italy and France. Chaos Solitons Fractals $134: 5$

15. Kermack WO, McKendrick AG (1932) Contributions to the mathematical theory of epidemics. II: the problem of endemicity. Proc R Soc Lond Ser A 138(834):55-83

16. Capasso V, Serio G (1978) A generalization of the KermackMcKendrick deterministic epidemic model. Math Biosci 42(1-2):43-61

17. Weiss HH (2013) The SIR model and the foundations of public health. Mater Mat 2013(3):1-17

18. Peng L et al (2020) Epidemic analysis of COVID-19 in China by dynamical modeling. arXiv:2002.06563

19. Ahmar AS, del Val EB (2020) SutteARIMA: short-term forecasting method, a case-Covid-19 and stock market in Spain. Sci Total Environ 729:138883

20. Al-qaness MAA et al (2020) Optimization method for forecasting confirmed cases of COVID-19 in China. J Clin Med 9(3):674

21. Anastassopoulou $C$ et al (2020) Data-based analysis, modelling and forecasting of the COVID-19 outbreak. PLoS ONE 15(3): 0230405

22. Chakraborty T, Ghosh I (2020) Real-time forecasts and risk assessment of novel coronavirus (COVID-19) cases: a data-driven analysis. Chaos Solitons Fractals 135:10

23. Chaudhry RM et al (2020) Coronavirus disease 2019 (COVID19): forecast of an emerging urgency in Pakistan. Cureus 12(5): 15 
24. Chen DG, Chen XG, Chen JK (2020) Reconstructing and forecasting the COVID-19 epidemic in the United States using a 5-parameter logistic growth model. Glob Health Res Policy $5(1): 7$

25. Chimmula VKR, Zhang L (2020) Time series forecasting of COVID-19 transmission in Canada using LSTM networks. Chaos Solitons Fractals 135:6

26. Chintalapudi N, Battineni G, Amenta F (2020) COVID-19 virus outbreak forecasting of registered and recovered cases after sixty day lockdown in Italy: a data driven model approach. J Microbiol Immunol Infect 53(3):396-403

27. Kavadi DP, Patan R, Ramachandran M, Gandomi AH (2020) Partial derivative nonlinear global pandemic machine learning prediction of COVID-19. Chaos Solitons Fractals. https://doi.org/ 10.1016/j.chaos.2020.110056

28. Mousavi M, Salgotra R, Holloway D, Gandomi AH COVID-19 time series forecast using transmission rate and meteorological parameters as features. IEEE Comput Intell Mag (in press). https://doi.org/10.1109/mci.2020.3019895

29. Al-qaness MAA et al (2020) Optimization method for forecasting confirmed cases of COVID-19 in China. J Clin Med 9(3):15

30. Singh $S$ et al (2020) Development of new hybrid model of discrete wavelet decomposition and autoregressive integrated moving average (ARIMA) models in application to one month forecast the casualties cases of COVID-19. Chaos Solitons Fractals 135:8

31. Moftakhar L, Seif M, Safe MS (2020) Exponentially increasing trend of infected patients with COVID-19 in Iran: a comparison of neural network and ARIMA forecasting models. Iran J Public Health 49:92-100

32. Salgotra R et al (2020) Time series analysis and forecast of the COVID-19 pandemic in India using genetic programming. Chaos Solitons Fractals 138:109945

33. Salgotra R, Gandomi M, Gandomi AH (2020) Evolutionary modelling of the COVID-19 pandemic in fifteen most affected countries. Chaos Solitons Fractals. https://doi.org/10.1016/j. chaos.2020.110118

34. Salgotra R, Gandomi AH (in press) Time series analysis of the COVID-19 pandemic in Australia using genetic programming. In: Kose U et al (eds) Data science for COVID 19. Elsevier

35. Maleki $\mathrm{M}$ et al (2020) Time series modelling to forecast the confirmed and recovered cases of COVID-19. Travel Med Infect Dis 37:101742

36. Reno C et al (2020) Forecasting COVID-19-associated hospitalizations under different levels of social distancing in Lombardy and Emilia-Romagna, Northern Italy: results from an extended SEIR compartmental model. J Clin Med 9(5):11

37. Li Q, Feng W, Quan YH (2020) Trend and forecasting of the COVID-19 outbreak in China. J Infect 80(4):472-474

38. Qeadan $F$ et al (2020) Naive forecast for COVID-19 in Utah based on the South Korea and Italy models-the fluctuation between two extremes. Int J Environ Res Public Health 17(8):14

39. Fong SJ et al (2020) Finding an accurate early forecasting model from small dataset: a case of $2019-\mathrm{nCoV}$ novel coronavirus outbreak. Int J Interact Multimed Artif Intell 6(1):132-140

40. Tamang SK, Singh PD, Datta B (2020) Forecasting of Covid-19 cases based on prediction using artificial neural network curve fitting technique. Glob J Environ Sci Manag 6:53-64

41. Ren HY et al (2020) Early forecasting of the potential risk zones of COVID-19 in China's megacities. Sci Total Environ 729:8

42. Ji D et al (2020) Prediction for progression risk in patients with COVID-19 pneumonia: the CALL Score. Clin Infect Dis 71(6):1393-1399. https://doi.org/10.1093/cid/ciaa414

43. Ribeiro MHDM et al (2020) Short-term forecasting COVID-19 cumulative confirmed cases: perspectives for Brazil. Chaos Solitons Fractals 135:109853
44. Sujath R et al (2020) A machine learning forecasting model for COVID-19 pandemic in India. Stochast Environ Res Risk Assess 34:959-972

45. Abdulmajeed K, Adeleke M, Popoola LJD (2020) Online forecasting of Covid-19 cases in Nigeria using limited data. Data Brief 30:105683

46. Roosa $\mathrm{K}$ et al (2020) Real-time forecasts of the COVID-19 epidemic in China from February 5th to February 24th, 2020. Infect Dis Model 5:256-263

47. Parbat, D.J.C., Solitons and Fractals, A Python based Support Vector Regression Model for prediction of Covid19 cases in India. 2020: p. 109942

48. Perc $\mathrm{M}$ et al (2020) Forecasting Covid-19. Front Phys 8:127

49. Maleki M, Arellano-Valle RB (2017) Maximum a posteriori estimation of autoregressive processes based on finite mixtures of scale-mixtures of skew-normal distributions. J Stat Comput Simul 87(6):1061-1083

50. Maleki M, Nematollahi AJ, Technology TAS (2017) Autoregressive models with mixture of scale mixtures of Gaussian innovations. Iran J Sci Technol Trans A Sci 41(4):1099-1107

51. Zarrin $P$ et al (2019) Time series models based on the unrestricted skew-normal process. J Stat Comput Simul 89(1):38-51

52. Maleki $\mathrm{M}$ et al (2017) A Bayesian approach to robust skewed autoregressive processes. Calcutta Stat Assoc Bull 69(2):165-182

53. Hajrajabi A, Maleki MJ (2019) Nonlinear semiparametric autoregressive model with finite mixtures of scale mixtures of skew normal innovations. J Appl Stat 46(11):2010-2029

54. Sprinthall RC, Fisk ST (1990) Basic statistical analysis. Prentice Hall, Englewood Cliffs

55. Roy S, Bhunia GS, Shit PK (2020) Spatial prediction of COVID19 epidemic using ARIMA techniques in India. Model Earth Syst Environ. https://doi.org/10.1007/s40808-020-00890-y

56. Alzahrani SI et al (2020) Forecasting the spread of the COVID-19 pandemic in Saudi Arabia using ARIMA prediction model under current public health interventions. J Infect Public Health 13:914-919

57. Kufel TJ (2020) ARIMA-based forecasting of the dynamics of confirmed Covid-19 cases for selected European countries. Equilib Q J Econ Econ Policy 15(2):181-204

58. Singh $S$ et al (2020) Development of new hybrid model of discrete wavelet decomposition and autoregressive integrated moving average (ARIMA) models in application to one month forecast the casualties cases of COVID-19. Chaos Solitons Fractals 135:109866

59. Smith BAJIDM (2020) A novel IDEA: the impact of serial interval on a modified-incidence decay and exponential adjustment (m-IDEA) model for projections of daily COVID-19 cases. Infect Dis Model 5:346-356

60. Bengio $\mathrm{Y}$ et al (2013) Representation learning: a review and new perspectives. IEEE Trans Pattern Anal Mach Intell 35(8): 1798-1828

61. Schmidhuber JJ (2015) Deep learning in neural networks: an overview. Neural Netw 61:85-117

62. LeCun Y, Bengio Y, Hinton GJ (2015) Deep learning. Nature 521(7553):436-444

63. Ayyoubzadeh SM et al (2020) Predicting COVID-19 incidence through analysis of Google trends data in Iran: data mining and deep learning pilot study. JMIR Public Health Surveill 6(2):e18828

64. Freedman DA (2009) Statistical models: theory and practice. Cambridge University Press, Cambridge

65. Cook RD, Weisberg SJ (1982) Criticism and influence analysis in regression. Sociol Methodol 13:313-361

66. Velásquez RMA, Lara JVMJC (2020) Forecast and evaluation of COVID-19 spreading in USA with reduced-space Gaussian process regression. Chaos Solitons Fractals 136:109924 
67. Almeshal AM et al (2020) Forecasting the spread of COVID-19 in Kuwait using compartmental and logistic regression models. Appl Sci 10(10):3402

68. Taylor SJ, Letham B (2018) Forecasting at scale. Am Stat 72(1):37-45

69. Taylor S, Letham B (2017) Prophet: automatic forecasting procedure. R package version 0.1. https://CRAN.R-project.org/pack age $=$ prophet

70. Ndiaye BM, Tendeng L, Seck D (2020) Analysis of the COVID19 pandemic by SIR model and machine learning technics for forecasting. arXiv:2004.01574

71. Banzhaf W et al (1998) Genetic programming. Springer, Berlin

72. Jalalkamali A et al (2015) Application of several artificial intelligence models and ARIMAX model for forecasting drought using the Standardized Precipitation Index. Int J Environ Sci Technol 12(4):1201-1210

73. Wilamowski BMJIIEM (2009) Neural network architectures and learning algorithms. IEEE Ind Electron Mag 3(4):56-63

74. Hethcote HW (1989) Three basic epidemiological models. In: Levin SA, Hallam TG, Gross LJ (eds) Applied mathematical ecology. Springer, Berlin, pp 119-144

75. Brauer F, Castillo-Chavez C (2012) Mathematical models in population biology and epidemiology, vol 2. Springer, Berlin

76. Nunn C, Altizer S, Altizer SM (2006) Infectious diseases in primates: behavior, ecology and evolution. Oxford University Press, Oxford

77. Gao S et al (2007) Analysis of an SIR epidemic model with pulse vaccination and distributed time delay. J Biomed Biotechnol 2007:64870. https://doi.org/10.1155/2007/64870
78. Rahimi I, Gandomi AH, Chen F et al (2020) Analysis and prediction of COVID-19 using SIR, SEIR, and machine learning models: Australia, Italy, and UK Cases, 13 October 2020, PREPRINT (version 1) available at Research Square. https://doi.org/ 10.21203/rs.3.rs-85513/v1

79. Bartlett MS (1957) Measles periodicity and community size. J R Stat Soc Ser A 120(1):48-70

80. Glover F, Laguna M (1998) Tabu search. Handbook of combinatorial optimization. Springer, Boston, MA, pp 2093-2229

81. Poojari CA, Beasley JE (2009) Improving benders decomposition using a genetic algorithm. Eur J Oper Res 199(1):89-97

82. Blum C, Roli AJ (2003) Metaheuristics in combinatorial optimization: overview and conceptual comparison. ACM Comput Surv 35(3):268-308

83. Makridakis S, Hibon MJ (1997) ARMA models and the BoxJenkins methodology. J Forecast 16(3):147-163

84. Ciregan D, Meier U, Schmidhuber J (2012) Multi-column deep neural networks for image classification. In: 2012 IEEE conference on computer vision and pattern recognition. IEEE

85. Krizhevsky A, Sutskever I, Hinton GE (2017) Imagenet classification with deep convolutional neural networks. Commun ACM 60(6):84-90. https://doi.org/10.1145/3065386

Publisher's Note Springer Nature remains neutral with regard to jurisdictional claims in published maps and institutional affiliations. 